Bond University

Research Repository

\title{
Effect of saffron supplementation on symptoms of depression and anxiety: a systematic review and meta-analysis
}

Marx, Wolfgang; Lane, Melissa; Rocks, Tetyana; Ruusunen, Anu; Loughman, Amy; Lopresti, Adrian; Marshall, Skye; Berk, Michael; Jacka, Felice; Dean, Olivia May

Published in:

Nutrition Reviews

DOI:

10.1093/nutrit/nuz023

Licence:

Free to read

Link to output in Bond University research repository.

Recommended citation(APA):

Marx, W., Lane, M., Rocks, T., Ruusunen, A., Loughman, A., Lopresti, A., Marshall, S., Berk, M., Jacka, F., \& Dean, O. M. (2019). Effect of saffron supplementation on symptoms of depression and anxiety: a systematic review and meta-analysis. Nutrition Reviews, 77(8), 557-571. [nuz023]. https://doi.org/10.1093/nutrit/nuz023

\section{General rights}

Copyright and moral rights for the publications made accessible in the public portal are retained by the authors and/or other copyright owners and it is a condition of accessing publications that users recognise and abide by the legal requirements associated with these rights.

For more information, or if you believe that this document breaches copyright, please contact the Bond University research repository coordinator. 


\title{
Effect of saffron supplementation on symptoms of depression and anxiety: a systematic review and meta-analysis
}

Wolfgang Marx, Melissa Lane, Tetyana Rocks, Anu Ruusunen, Amy Loughman, Adrian Lopresti, Skye Marshall, Michael Berk, Felice Jacka, and Olivia M. Dean

\begin{abstract}
Context: Saffron (Crocus sativus L.) has gained interest as a potential treatment in psychiatry. Objective: This systematic review and meta-analysis sought to investigate the effect of saffron supplementation, as both an adjunctive therapy and monotherapy, on symptoms of depression and anxiety in clinical and general populations compared with pharmacotherapy or placebo. Data sources: Using the PRISMA guidelines, a systematic literature review of randomized controlled trials was conducted. Data extraction: A meta-analysis was conducted to determine treatment effect. Risk of bias was assessed using the Jadad scale. Results: Twentythree studies were included. Saffron had a large positive effect size when compared with placebo for depressive symptoms $(g=0.99, P<0.001)$ and anxiety symptoms ( $g=0.95, P<0.006)$. Saffron also had a large positive effect size when used as an adjunct to antidepressants for depressive symptoms ( $g=1.23, P=0.028$ ). Egger's regression test found evidence of publication bias. Conclusions: Saffron could be an effective intervention for symptoms of depression and anxiety; however, due to evidence of publication bias and lack of regional diversity, further trials are required. PROSPERO registration CRD42017070060.
\end{abstract}

\section{INTRODUCTION}

Saffron (Crocus sativus L.) and its constituents, including crocin, crocetin, and safranal, may have a positive effect on several neurobiological mechanisms implicated in mental illness. ${ }^{1-3}$ In animal models, whole saffron and implicated compounds have been reported to modulate pathways related to neurotransmitters, immune regulation, inflammation, oxidative stress, the hypothalamus-pituitary-adrenal (HPA) axis, and neurotrophins. ${ }^{4}$ For example, in rats, the administration of a saffron extract dose-dependently increased brain concentrations of dopamine and, at high doses, increased glutamate levels. ${ }^{5}$ In another study, the administration of crocin modulated serotonergic activity in rats exposed to the nonselective serotonin receptor agonist

Affiliation: W. Marx, M. Lane, T. Rocks, A. Ruusunen, A. Loughman, and F. Jacka are with the Food \& Mood Centre, IMPACT SRC, School of Medicine, Deakin University, Geelong, Australia. M. Berk and O.M. Dean are with the IMPACT SRC, School of Medicine, Barwon Health, Deakin University, Geelong, Australia. A. Ruusunen is with the Department of Psychiatry, Kuopio University Hospital, Kuopio, Finland, and the Institute of Public Health and Clinical Nutrition, University of Eastern Finland, Kuopio, Finland. A. Lopresti is with the School of Psychology and Exercise Science, Murdoch University, Perth, Western Australia, Australia. S. Marshall is with the Faculty of Health Sciences \& Medicine, Bond University, Robina, Queensland, Australia. M. Berk is with the Department of Psychiatry, University of Melbourne, Melbourne, Australia, and Orygen, the National Centre of Excellence in Youth Mental Health, Centre for Youth Mental Health and the Florey Institute for Neuroscience and Mental Health, University of Melbourne, Melbourne, Australia. F. Jacka is with the Centre for Adolescent Health, Murdoch Children's Research Institute, Victoria, Australia, and the Black Dog Institute, New South Wales, Australia. O.M. Dean is with the Florey Institute for Neuroscience and Mental Health, and the Department of Psychiatry, University of Melbourne, Parkville, Australia.

Correspondence: W. Marx, Food \& Mood Centre, 285 Ryrie St, 3220 Victoria, Australia. E-mail: wolf.marx@deakin.edu.au.

Key words: depression, herbal medicine, inflammation, mental health, mood disorder, nutraceutical, saffron, treatment.

(c) The Author(s) 2019. Published by Oxford University Press on behalf of the International Life Sciences Institute. All rights reserved. For permissions, please e-mail: journals.permissions@oup.com. 
meta-chlorophenylpiperazine, lowering obsessive-like behaviors. ${ }^{6}$ Saffron constituents also have antiinflammatory, immune-regulating, and antioxidant properties, including the modulation of endogenous antioxidant enzymes. ${ }^{7-11}$ In addition, studies using animal stress models have confirmed that saffron modulates HPA axis activity by reducing plasma corticosterone concentrations. ${ }^{12,13}$ Finally, support for the neuroprotective effects of saffron and its constituent, crocin, is derived from studies demonstrating positive effects on the neurotrophin, brain-derived neurotrophic factor (BDNF) in animal stress models. ${ }^{14,15}$

Two previous meta-analyses have reported saffron supplementation to improve measures of depression in clinical trials. ${ }^{16,17}$ Previous meta-analyses on this topic, however, have only examined the effects of saffron on depression in clinical populations with mild to major depression. ${ }^{16,17}$ Furthermore, since the publication of these reviews, several published intervention studies have been conducted in both depression and other mental illnesses. Therefore, using the Preferred Reporting Items for Systematic Reviews and Metaanalyses (PRISMA) guidelines, this systematic review and meta-analysis of randomized controlled trials (RCTs) aims to examine the transdiagnostic effects of saffron supplementation (as a stand-alone or adjunctive intervention) on symptoms of mental illness in both clinical and general populations compared with pharmacotherapy or placebo.

\section{METHODS}

\section{Literature search}

This review was written in accordance with the PRISMA (Preferred Reporting Items for Systematic reviews and Meta-Analyses) statement (see Appendix S1 in the Supporting Information online $)^{18}$ and was prospectively registered on PROSPERO (CRD42017070060). A search strategy was developed based on the research question (Table 1). Studies were identified using the following databases: Medline (Pubmed), PsychInfo, Embase, the Cochrane Library, and CINAHL. Search terms were related to saffron and its constituents ("saffron" OR "crocus" OR "crocin" OR "crocetin") and mental health outcomes ("depression" OR "depressive disorder" OR "anxiety disorders" OR "affective symptoms" OR anxi* OR mood OR "psychological symptoms" OR "psychological distress"). The search strategy identified articles published since journal inception up to March 2018.

Studies were required to be RCTs, including crossover studies; be published in English; include measures of mental health (eg, depression, low mood, depressive symptoms, emotional problems, and anxiety); and use any component of saffron (eg, whole saffron, standardized extracts, parts of saffron, or specific saffron-derived compounds). Interventions that included saffron in combination with standard medications were included; however, combined interventions with other novel ingredients were excluded. No limit on age or population was included, and the comparator could be either placebo or pharmacotherapy.

\section{Data extraction}

Two authors independently screened individual studies in duplicate, with disagreements resolved by consensus or a third author. Using a standardized and piloted extraction form, the following parameters were extracted from included studies: author/date, study design, sample size, total study period, population characteristics (including age, sex, and comorbidities), intervention characteristics (including dose, type, and duration of exposure, concomitant treatment), length of follow-up, adverse events, and mental health-related outcomes, including measures of depression, anxiety, mood. Outcomes not related to mental health were not extracted for this review.

\section{Assessment of study risk of bias}

Risk of bias was assessed using the Jadad scale, a 5-item tool that assesses risk of bias due to randomization, blinding, and follow-up. ${ }^{19}$ Studies can receive a score between 0 and 5, with a higher score indicating a lower risk of bias.

\section{Statistical analyses}

The meta-analyses were conducted in Comprehensive Meta-Analysis $3.0^{20}$ using a DerSimonian-Laird random-effects model ${ }^{21}$ to account for heterogeneity between studies. The primary outcome was the effect of saffron on total depressive symptoms in comparison with placebo control conditions. The effect of saffron on symptoms of depression as an adjunctive to medication and as a stand-alone intervention compared with medication was also computed as a secondary outcome. The effects of saffron on symptoms of anxiety compared with placebo, medication, and as an adjunctive intervention were also computed when sufficient data were available. Mean change scores in symptoms for saffron and control conditions were compared using randomeffects meta-analyses to compute effect size of saffron compared with control condition as Hedges' $g$ (with $95 \% \mathrm{CI}$ ). For studies that used $>1$ measure of the same 
outcome, the measure identified as the primary outcome by the respective study was used in meta-analyses. Otherwise, an average change score across all measures was computed and used in the pooled analysis. In multi-arm trials that had $>1$ active saffron condition, a pooled effect was computed across all saffron arms compared with the nonsaffron comparator conditions. To examine the possibility of publication bias affecting results, Egger's $t$ test was conducted. Finally, to further account for the potential impact of publication bias, a Duval and Tweedie's trim-and-fill analysis was applied to recalculate effect sizes after statistically accounting for outlier studies.

Additionally, subgroup analyses were conducted to examine the 1) effects of saffron in comparison with antidepressant medications and 2) the effect of saffron on depression when used as an adjunctive treatment alongside antidepressants (ie, studies comparing saffron to placebo in samples already taking or commencing antidepressant medications).

Heterogeneity between studies was examined using Cochran's $Q$ (with $P$ value) and $I^{2}$ estimates. These statistics estimate the extent to which statistical heterogeneity between studies has arisen from genuine interstudy differences rather than chance.

\section{RESULTS}

\section{Study selection}

As represented in Figure 1, the search strategy resulted in 310 de-duplicated studies that were screened to identify 23 eligible studies for inclusion (see Table $2^{22-44}$ ).

\section{Study characteristics}

A total of 1237 participants were enrolled in the included studies, with 30-128 participants in each study. Trials ran 4-12 weeks with 6 weeks being the most common trial length $(n=9 / 23)$. Seventeen studies investigated saffron monotherapy ( $\mathrm{n}=11$ studies) or saffron as an adjunctive pharmacotherapy compared with placebo ( $\mathrm{n}=6$ studies). Six studies compared saffron monotherapy with an antidepressant medication (including fluoxetine, ${ }^{23,32,40,42}$ imipramine, ${ }^{25}$ and citalo$\operatorname{pram}^{27}$ ) No study investigated saffron as an adjunct to psychotherapy. Nineteen studies included participants with either clinical diagnosis of mental illness or clinical symptoms of mental illness using a validated tool. The average age of participants was 39 years, with a range of 14-57 years. Most studies included a mix of sexes, with 5 studies including only females and 1 study including only males. Most studies were conducted in Iran $(\mathrm{n}=$ 21/23), with 2 studies conducted in Australia. ${ }^{34,35}$
Table 1 PICOS criteria for inclusion and exclusion of studies

\begin{tabular}{ll}
\hline Parameter & \multicolumn{1}{c}{ Criteria } \\
\hline Population & $\begin{array}{c}\text { Human participants, both clinically diagnosed } \\
\text { with a mental illness and otherwise } \\
\text { Intervention }\end{array}$ \\
$\begin{array}{c}\text { Saffron supplementation. Both whole saffron } \\
\text { or as an extract } \\
\text { Clacebo or standard antidepressant } \\
\text { medication }\end{array}$ \\
Outcomes & Symptoms of mental illness, adverse events \\
\hline
\end{tabular}

Thirteen studies were conducted by the same research group. Risk of bias across most studies was low, with 20 studies receiving a score of 4 or 5 (out of 5) on the Jadad Scale (see Appendix S2 in the Supporting Information online).

\section{Interventions}

Most studies $(n=19 / 23)$ used a dose of $30 \mathrm{mg}$ per day of saffron. Ten studies used the stigma of saffron, 4 studies used saffron petals, 3 used crocin only, and the remaining studies used either a whole powder or did not provide further details. In 9 studies, the intervention was standardized to contain either crocin, safranal, or lepticrosalides (a measure of bioactive compounds present in saffron, including safranal and crocin isomers).

\section{Outcome measures}

Depressive symptoms $(n=22 / 23)$ were more commonly investigated than anxiety symptoms $(n=8 / 23)$. Depression and/or anxiety symptoms were measured using the Hamilton Rating Scale for Depression or Anxiety, Beck Depression Inventory, Depression Anxiety Stress Scale (DASS) 21, or Revised Child Anxiety and Depression Scale (RCADS; youth and parent versions). One study assessed mood using the Profile of Mood States (POMS) and Positive and Negative Affect Schedule (PANAS) 21 tools.

\section{Study results}

Effects of saffron on depressive symptoms. Randomeffects meta-analysis found a significant and large positive effect size for saffron reducing symptoms of depression in comparison with placebo $(g=0.99$, $95 \% \mathrm{CI}=0.61-1.37, \mathrm{n}=14$ studies, $\mathrm{n}=716$ participants, $P<0.001$; Figure $2^{22-27,29-31,33-40,42,44}$ ). An additional study that could not be entered into the metaanalysis due to insufficient available data also found that saffron significantly improved depressive symptoms compared with placebo $(P<0.0001) .{ }^{43}$ There was significant heterogeneity across the study data $(Q=$ 


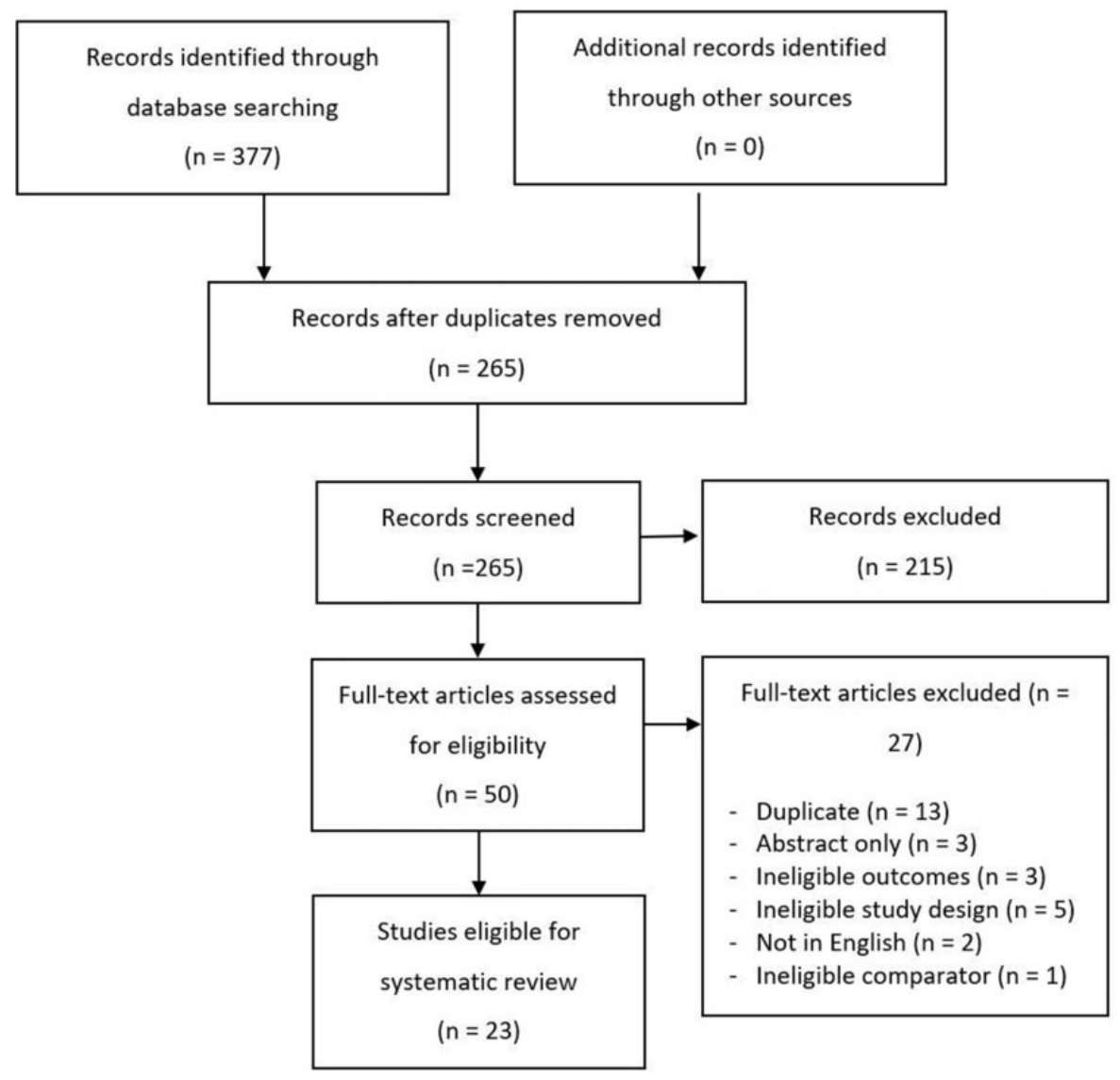

Figure 1 Flow diagram of the literature search process.

71.8, $\left.P<0.001, I^{2}=81.9 \%\right)$. Egger's regression test found strong evidence of publication bias (Intercept $=$ $6.99, P=0.007)$. The funnel plot of publication bias is displayed in Appendix S3 in the Supporting Information online. Correcting for publication bias using a trim-andfill analysis increased the effect size of saffron supplementation $(g=1.14,95 \% \mathrm{CI}=0.74-1.52)$.

When compared with antidepressants medications, there was no significant difference between saffron and medications $(g=-0.17,95 \% \mathrm{CI}=0.50-0.17$, $\mathrm{n}=5$ studies, $\mathrm{n}=210$ participants, $P=0.33$; Figure $2^{22-27,29-31,33-40,42,44}$ ). There was a low degree of heterogeneity across the study data $(Q=6.16, P=$ $\left.0.19, I^{2}=35.1 \%\right)$. One study for which sufficient information was not provided to be included in the metaanalysis also found no significant difference in depressive symptoms between saffron and medication $(P=0.61) .{ }^{32}$

Meta-analysis of studies that investigated the effect of saffron as an adjunct to pharmacotherapy (ie, in studies comparing saffron to placebo in people taking antidepressants) reported a large significant benefit from saffron supplementation $(\mathrm{n}=4$ trials, $\mathrm{n}=144$ participants, $g=1.23,95 \% \mathrm{CI}=0.13-2.33, P=0.028$, $\left.Q=27.62, I^{2}=89.1 \%\right)$. One study that could not be included in the meta-analysis reported no significant difference in depressive symptoms between the adjunctive group (fluoxetine and saffron) and control (fluoxetine and placebo $)(P=0.560) .{ }^{41}$

Effects of saffron on symptoms of anxiety. Figure $3^{28,34-37,4428,34-37,44}$ displays the effects of saffron monotherapy and adjunctive therapy on symptoms of anxiety across 6 RCTs compared with placebo ( $\mathrm{n}=375$ participants). Random-effects meta-analysis found a large positive effect size for saffron on reducing symptoms of anxiety in comparison with placebo control conditions $(g=0.95,95 \% \mathrm{CI}=0.27-1.63, P<0.006)$. There was significant heterogeneity across the study data $\left(Q=44.38, P<0.001, I^{2}=88.74 \%\right)$. Egger's regression test found strong evidence of publication bias (Intercept $=0.12, P=0.028$ ). Correcting for publication bias using a trim-and-fill analysis increased the effect size of saffron supplementation $(g=1.40,95 \% \mathrm{CI}=$ $0.60-2.150)$. There were insufficient studies to conduct a meta-analysis of the effect of saffron on anxiety in comparison with antidepressant medications or to conduct subgroup analysis of saffron as an adjunctive treatment. One study that investigated the effect of saffron for anxiety symptoms compared with citalopram found 


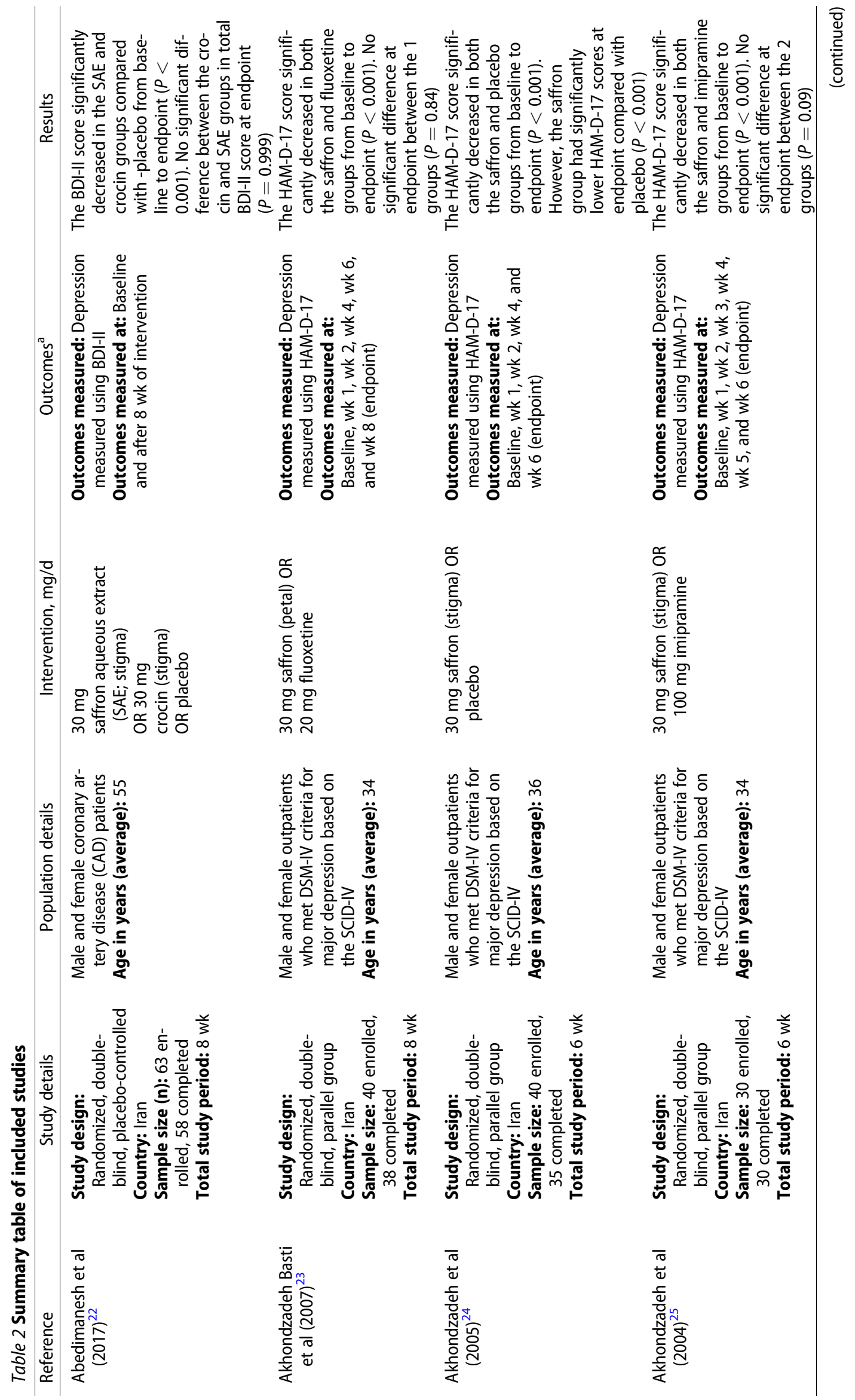




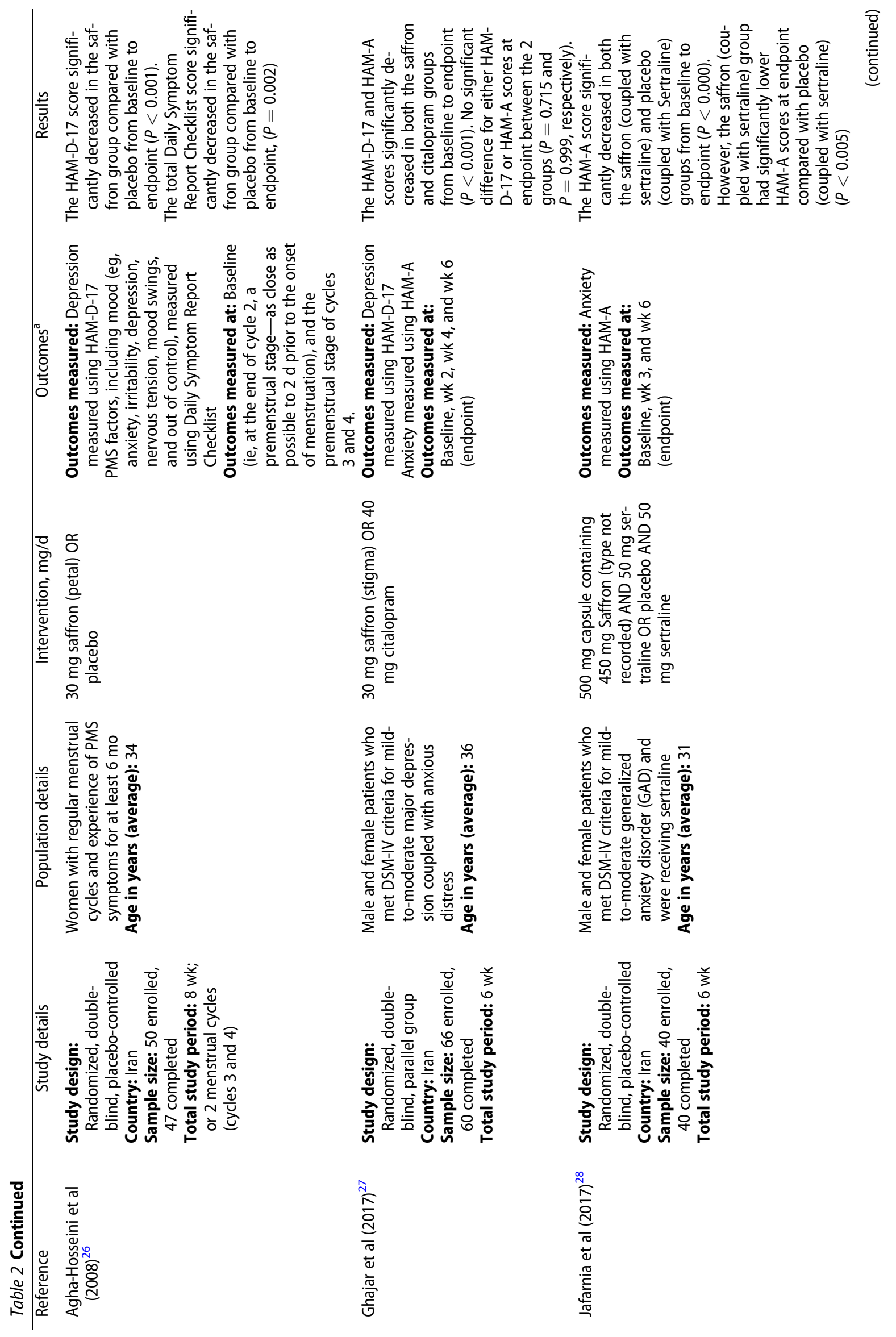




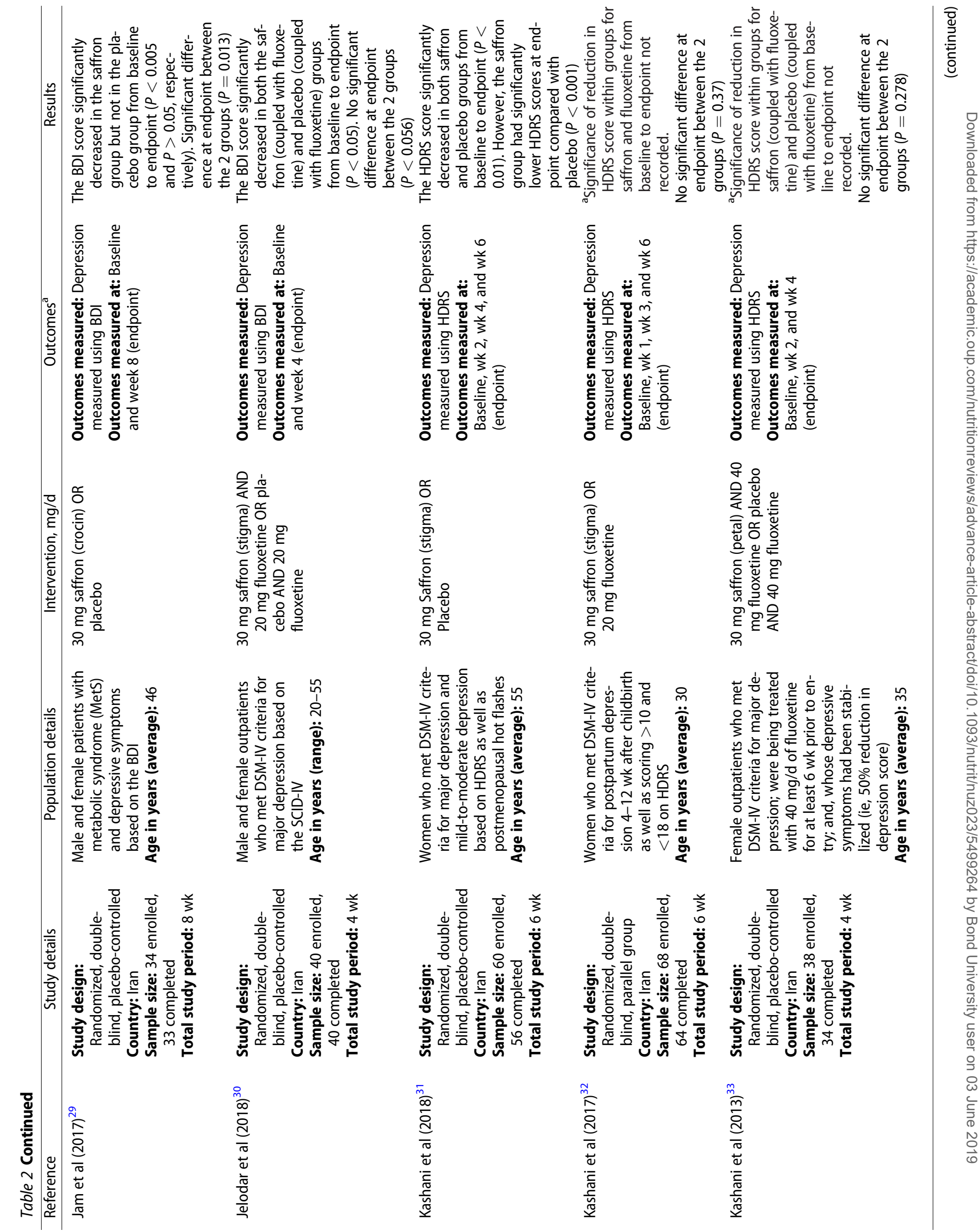




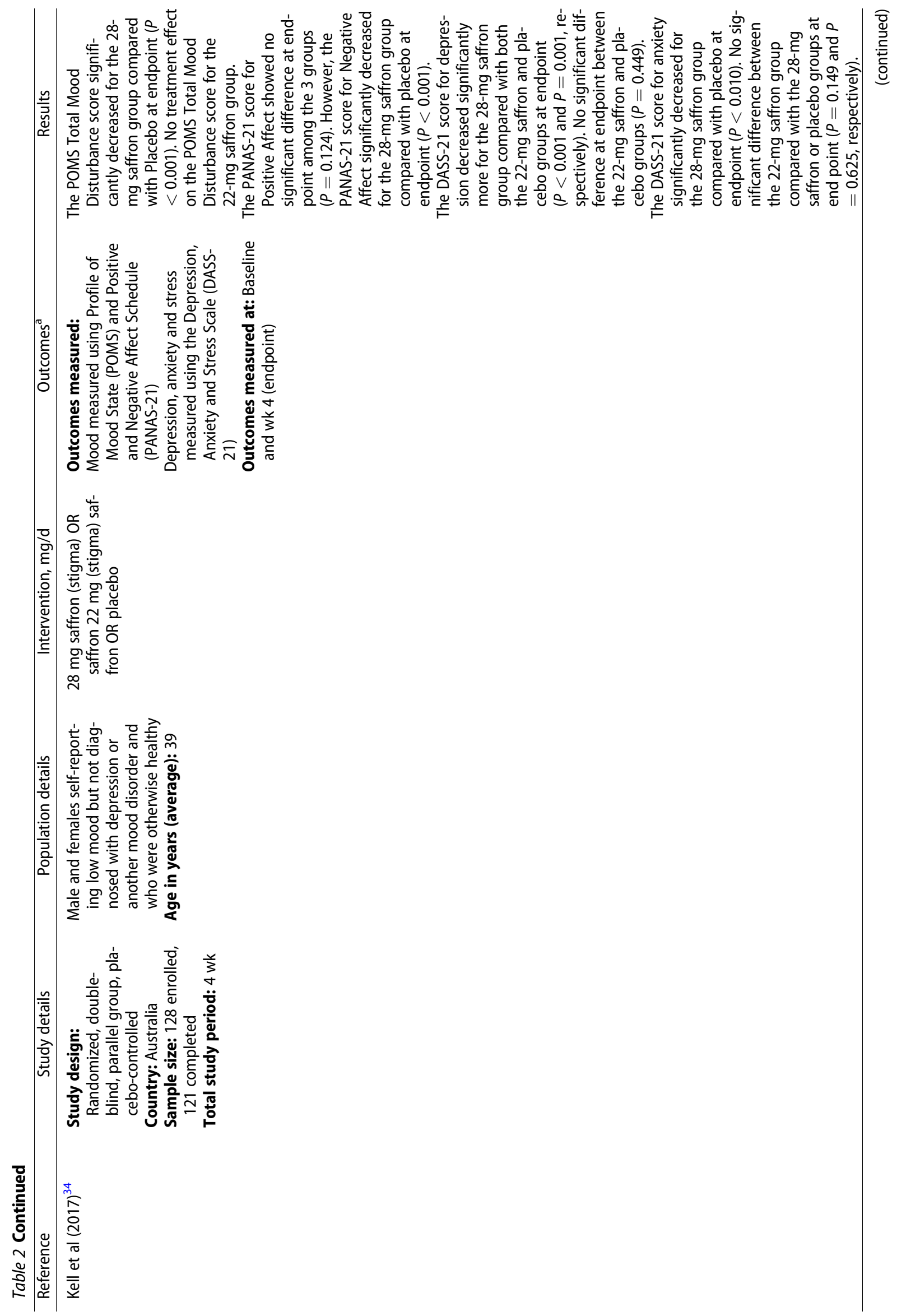




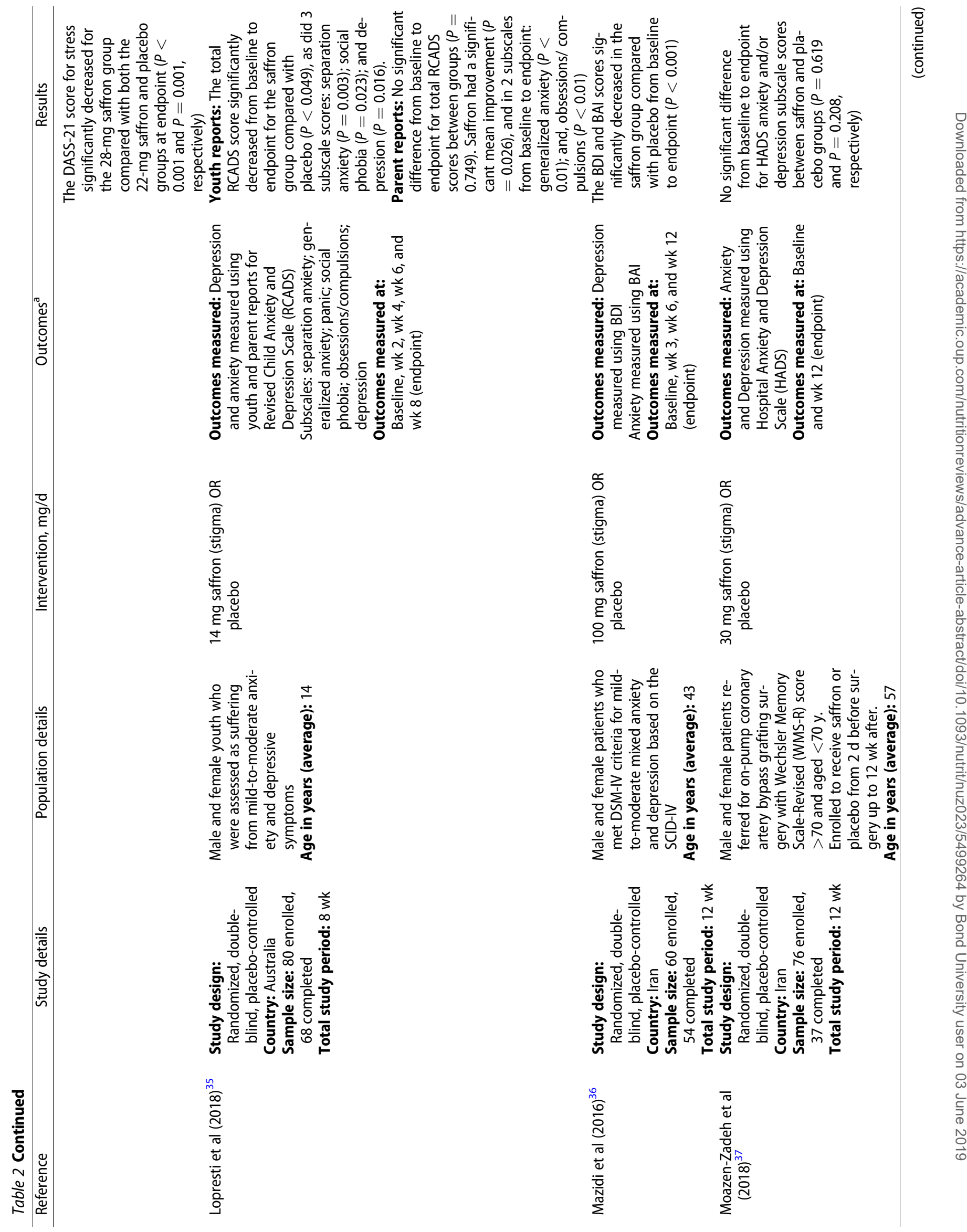




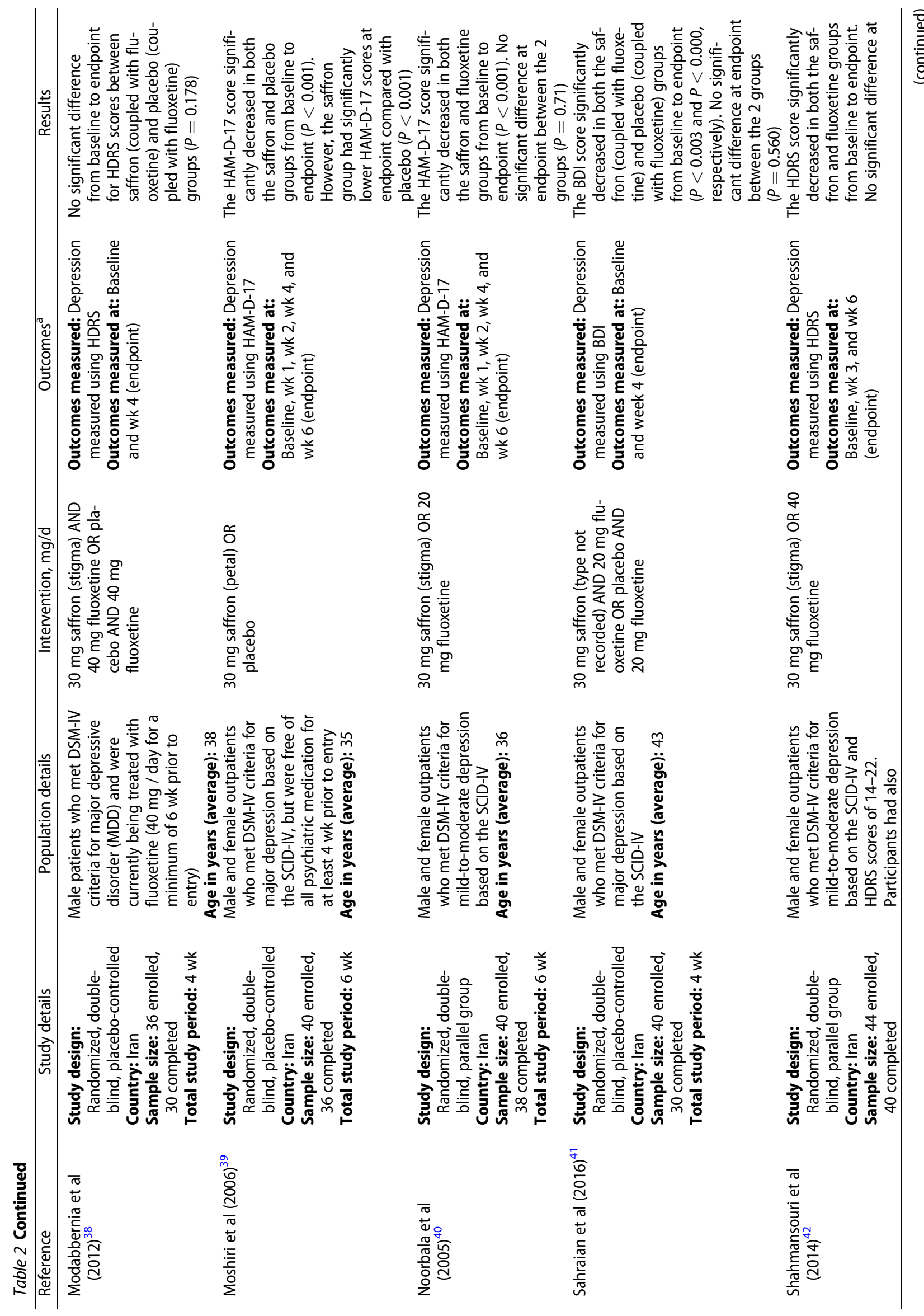




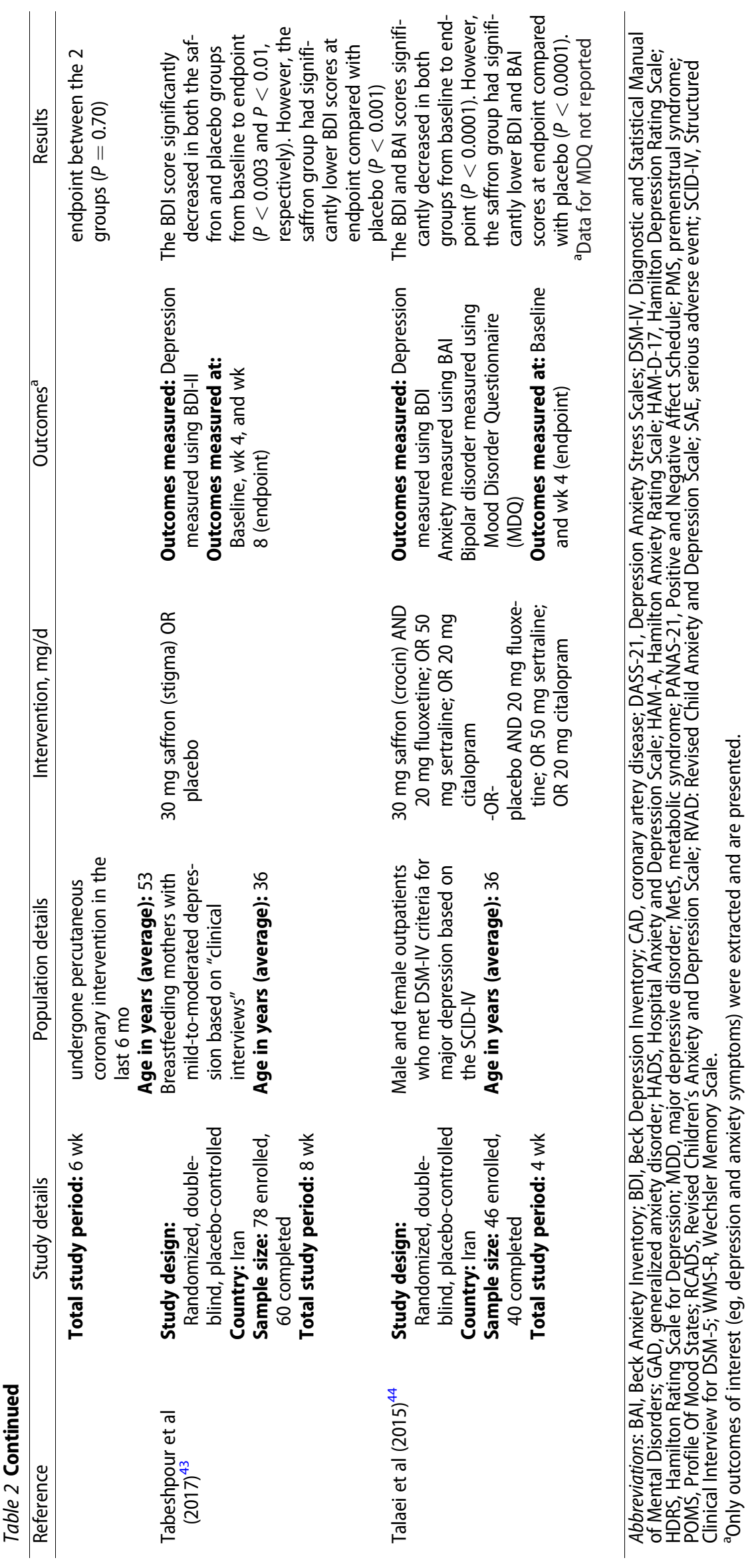


no significant difference between treatment groups $(P<0.001){ }^{27}$

Sensitivity analyses. Sensitivity analyses were performed to exclude 1 outlier study that could be influencing the overall findings. ${ }^{44}$ Exclusion of this study only slightly reduced the overall effect size of saffron on depressive symptoms compared with placebo $(g=0.84,95 \% \mathrm{CI}=$ $0.53-1.16, P<0.001)$, although large heterogeneity $\left(Q=45.45, P<0.001, I^{2}=73.8 \%\right)$ and significant publication bias was still evident in the remaining 13 studies (Egger's regression intercept $=4.957, P=0.043$ ).

When performing sensitivity analyses with the outlier study excluded for anxiety outcomes, the effects of saffron compared with placebo were reduced substantially, although overall results remained the same $(g=$ $0.48,95 \% \mathrm{CI}=0.25-0.70, P<0.001)$. There was also little indication of heterogeneity $(Q=3.39, P=0.50$, $I^{2}=0 \%$ ) or publication bias (Egger's regression intercept $=0.23, P=0.47$ ) in the remaining 5 studies, although this is likely due to standard tests for detecting heterogeneity/publication bias in meta-analysis being underpowered in this small number of studies.

Effects of saffron on additional outcomes. One study found a significant improvement in total score of mood disturbances $(P<0.001)$ and subscales of fatigue $(P=$ $0.007)$, tension $(P=0.025)$, vigor $(P=0.007)$, confusion $(P=0.001)$, negative affect $(P=0.001)$, and stress $(P<0.001)$ when saffron supplementation was compared with placebo. ${ }^{34}$ Another study found significant improvements in self-reported measures of internalizing symptoms $(P=0.049)$, separation anxiety $(P=$ $0.003)$, and social phobia $(P=0.023)$ compared with placebo. ${ }^{35}$ General mental health was improved in 1 study compared with placebo $(P<0.001),{ }^{44}$ and the emotional functioning subscale of a quality-of-life questionnaire also improved in a separate study after saffron supplementation compared with placebo $(P<0.001){ }^{22}$

\section{Adverse events}

Adverse events were reported for most studies $(n=21 / 23)$. Headache, nausea, anxiety, constipation, dry mouth, and appetite changes were the most commonly reported symptoms in both arms. No study found symptoms to occur in the saffron group at a statistically higher rate than in the placebo and medication groups.

\section{DISCUSSION}

This is the most comprehensive meta-analysis to evaluate the effects of saffron supplementation for depressive and anxiety symptoms. The inclusion of adult, adolescent, and subclinical depressive and anxiety populations, as well as the examination of both depression and anxiety outcomes, provides a greater understanding of the possible efficacy of saffron as an intervention across the common mental disorders.

The results of this meta-analysis are consistent with 2 prior meta-analyses that investigated saffron for mildto-major depression only. ${ }^{16,17}$ Saffron had a significant large positive effect size when compared with placebo for both depression $(g=0.99, P<0.001)$ and anxiety $(g$ $=0.95, P<0.006)$ outcomes. For depressive outcomes, saffron also had a significant large positive effect size when used as an adjunctive treatment to antidepressants $(g=1.23, P=0.028)$, and when compared with antidepressant medications, there was no significant difference between groups $(g=-0.17, P=0.33)$. The included studies demonstrate that saffron supplementation appears to be well-tolerated with few adverse events being reported by the included studies or by other authors. ${ }^{45}$ However, all studies to date have been of relatively short duration and with a limited sample size. Therefore, longer-term trials that are suitably powered are required to assess the safety of saffron supplementation.

In the context of standard interventions for depression and anxiety, the results from the placebocontrolled trials suggest an effect size that is considerably greater than standard pharmacotherapy, such as selective serotonin reuptake inhibitors (Cohen's $d=$ 0.30). ${ }^{46}$ However, the 5 trials directly comparing saffron with antidepressant medication found no statistically significant difference $(P=0.33)$. Due to the large effect sizes, the publication bias detected for both outcomes, and the majority of trials being conducted in 1 region, many by the same research group $(n=13 / 23)$, there is a need to replicate these results within other populations and in large, well-powered, rigorous trials before clinical recommendations are justified. Similarly, greater rigor regarding good manufacturing practice and standardization, dosage, and characterization of the active ingredients is needed.

In addition to the need to replicate existing studies, additional areas are recommended for future studies. First, the use of saffron for other mental illnesses is currently underexplored. Whereas 7 studies assessed anxiety symptoms, only 1 study included participants with a diagnosed anxiety disorder. Similarly, 1 study evaluated the safety and feasibility of saffron in schizophrenia, but no efficacy trials have been conducted to date. ${ }^{47}$ Furthermore, there is a need to explore comparative efficacy of the different components of saffron. One study found that saffron petal and stigma had equivalent efficacy in treating depressive symptoms in participants with major depression, but there is insufficient 


\begin{tabular}{|c|c|c|c|c|c|c|c|c|c|}
\hline \multirow[t]{2}{*}{ Study name } & \multirow{2}{*}{$\frac{\text { Group by }}{\text { Comparison }}$} & \multicolumn{8}{|c|}{ Statistics for each study } \\
\hline & & $\begin{array}{l}\text { Hedges's } \\
\mathbf{g}\end{array}$ & $\begin{array}{l}\text { Standard } \\
\text { error }\end{array}$ & Variance & $\begin{array}{l}\text { Lower } \\
\text { Imitt }\end{array}$ & $\begin{array}{l}\text { Upper } \\
\text { limit }\end{array}$ & z-Value & p-Value & Total \\
\hline Akhondzadeh Bastiet al. 2007 & Medication & -0.325 & 0.312 & 0.097 & -0.937 & 0.286 & -1.043 & 0.297 & 40 \\
\hline Akhondzadeh et al. 2004 & Medication & 0.606 & 0.364 & 0.132 & -0.107 & 1.319 & 1.666 & 0.096 & 30 \\
\hline Ghajar et al. 2016 & Medication & -0.270 & 0.256 & 0.066 & -0.772 & 0.232 & -1.054 & 0.292 & 60 \\
\hline Noorbala et al. 2005 & Medication & -0.517 & 0.315 & 0.099 & -1.135 & 0.101 & -1.639 & 0.101 & 40 \\
\hline \multirow[t]{2}{*}{ Shahmansouri et al. 2013} & Medication & -0.153 & 0.310 & 0.096 & -0.761 & 0.456 & -0.492 & 0.623 & 40 \\
\hline & Medication & -0.166 & 0.171 & 0.029 & -0.501 & 0.169 & -0.970 & 0.332 & 210 \\
\hline Abedimanesh et al. 2017 & Placebo & 0.678 & 0.279 & 0.078 & 0.131 & 1.225 & 2.430 & 0.015 & 58 \\
\hline Agha-Hosseini et al. 2008 & Placebo & 2.602 & 0.381 & 0.145 & 1.855 & 3.349 & 6.828 & 0.000 & 50 \\
\hline Akhondzadeh et al. 2005 & Placebo & 1.484 & 0.352 & 0.124 & 0.795 & 2.173 & 4.221 & 0.000 & 40 \\
\hline Jam et al. 2017 & Placebo & 0.586 & 0.347 & 0.121 & -0.095 & 1.267 & 1.687 & 0.092 & 33 \\
\hline Jelodar et al 2018 & Placebo & 1.009 & 0.330 & 0.109 & 0.362 & 1.655 & 3.058 & 0.002 & 40 \\
\hline Kashani et al 2013 & Placebo & 0.363 & 0.338 & 0.114 & -0.299 & 1.025 & 1.075 & 0.283 & 34 \\
\hline Kashani et al. 2018 & Placebo & 0.739 & 0.273 & 0.074 & 0.205 & 1.273 & 2.710 & 0.007 & 56 \\
\hline Kell et al. 2017 & Placebo & 0.684 & 0.208 & 0.043 & 0.276 & 1.092 & 3.288 & 0.001 & 121 \\
\hline Lopresi et al. 2018 & Placebo & 0.569 & 0.233 & 0.054 & 0.112 & 1.026 & 2.439 & 0.015 & 75 \\
\hline Mazidi et al 2016 & Placebo & 0.111 & 0.270 & 0.073 & -0.419 & 0.640 & 0.410 & 0.682 & 54 \\
\hline Moazen-Zadeh et al. 2017 & Placebo & 0.370 & 0.296 & 0.087 & -0.210 & 0.949 & 1.251 & 0.211 & 45 \\
\hline Modabbernia et al. 2012 & Placebo & 0.485 & 0.361 & 0.130 & -0.222 & 1.192 & 1.345 & 0.179 & 30 \\
\hline Moshiri et al. 2006 & Placebo & 1.722 & 0.365 & 0.133 & 1.007 & 2.437 & 4.719 & 0.000 & 40 \\
\hline \multirow[t]{2}{*}{ Talaei et al 2015} & Placebo & 3.236 & 0.476 & 0.227 & 2.302 & 4.170 & 6.793 & 0.000 & 40 \\
\hline & Placebo & 0.992 & 0.193 & 0.037 & 0.613 & 1.371 & 5.135 & 0.000 & 716 \\
\hline
\end{tabular}

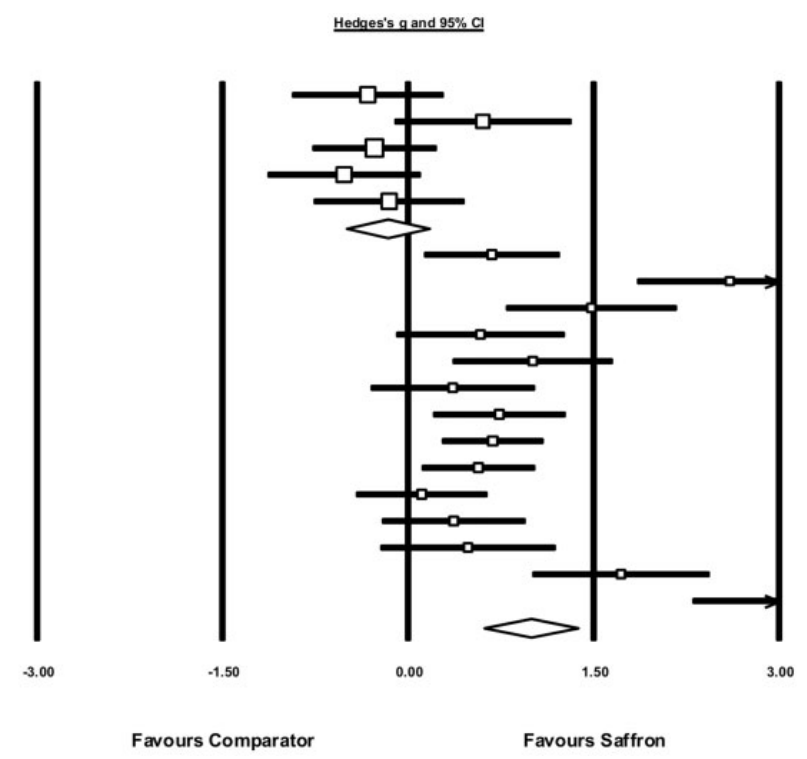

Figure 2 Meta-analysis showing effects of saffron on symptoms of depression in a) comparison with antidepressant medications, and b) comparison with placebo controls. Box size represents study weighting. Diamond represents overall effect size and $95 \%$ confidence intervals.

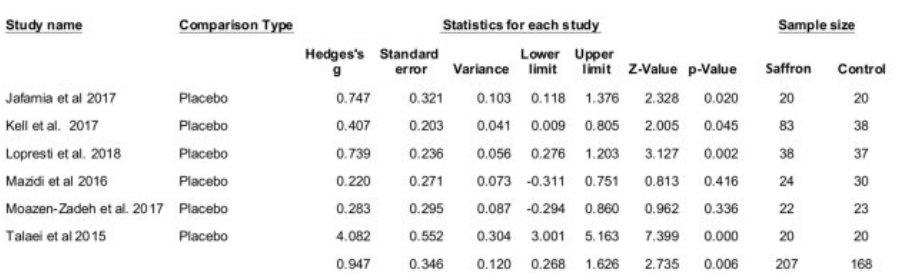

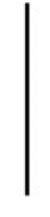

$-4.00$

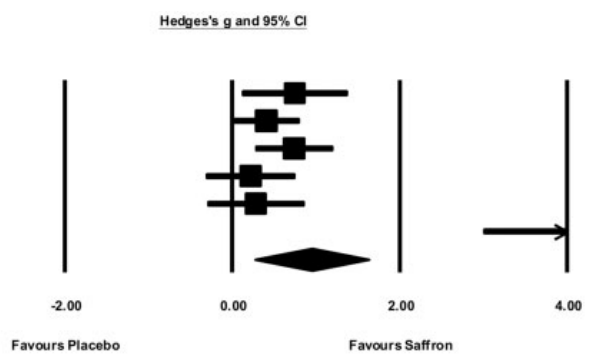

Favours Placebo

Figure 3 Meta-analysis of the effects of saffron on symptoms of anxiety. Box size represents study weighting. Diamond represents overall effect size and $95 \%$ confidence intervals.

literature to comment on particular components at this stage. ${ }^{23}$ Because of the high cost of saffron for culinary use, previous reviews have recommended the investigation of the efficacy of less expensive parts of saffron (eg, petal and corm). ${ }^{4}$ However, because of the small therapeutic dosage used in current studies, the cost of saffron formulations is unlikely to be considerably higher than for other nutraceutical interventions.

Preclinical studies suggest that compounds contained within saffron have a range of potentially relevant mechanisms of action, including antioxidant and anti-inflammatory properties, and are able to modulate BDNF expression and the HPA axis. ${ }^{4}$ However, these pathways have been largely unexplored within clinical trials. Hence, there is a need to measure markers of relevant pathways within future clinical trials to explore the association between changes in these biological markers and clinical symptoms. Furthermore, as demonstrated by Rapaport et al, ${ }^{48}$ who reported that treatment response to n-3 (eicosapentaenoic acid) was greater in those with higher inflammation at baseline, measuring relevant biomarkers may provide valuable information regarding specific populations and participant stratification to determine who is likely to respond to saffron treatment. Related to this is the need for dose-finding studies to establish the optimal dosing regimen. Most studies have used $30 \mathrm{mg}$ of saffron per day; however, it is unclear whether there are other regimens that may improve the therapeutic effect.

\section{CONCLUSION}

In summary, the existing evidence suggests a potential for saffron to be an efficacious intervention for symptoms of depression and anxiety. However, the strength of this conclusion is moderated by the lack of largescale trials and risk of publication bias among the many small-scale pilot trials published on this topic. Indeed, the large effect sizes reported for some studies require replication in further clinical trials that address methodological limitations and the lack of regional diversity before clinical recommendations can be made. Trials that 
explore the mechanisms of action of saffron, dosage, active ingredients, long-term safety, and the efficacy in diagnosed mental illnesses are also required.

\section{Acknowledgments}

The authors would like to acknowledge Dr Joe Firth and Professor Jerome Sarris for their contribution on aspects of the analyses and interpretation for the initial version of the manuscript.

Author contributions. F.J. and O.M.D. are co-senior authors. All authors contributed to the final manuscript. W.M. led all stages of the manuscript. M.L. and A. Loughman conducted the data extraction. S.M. conduct the risk-of-bias assessment. T.R., M.L., and A.R. extracted the data from included studies. A. Lopresti contributed to the mechanisms discussion and background.

Funding. No funding was provided for the development of this manuscript. W.M. is supported by a Deakin University research fellowship. T.R. is supported by a Fernwood Foundation Fellowship. A.R. is supported by a Deakin University research fellowship. A. Loughman is supported by the Wilson Foundation. M.B. is supported by a National Health and Medical Research Council (NHMRC) Senior Principal Research Fellowship (grant number 1059660). F.J. is supported by an NHMRC Career Development Fellowship (1108125). O.M.D. is supported by a Career Development Fellowship (APP1145634) from the NHMRC.

Declaration of interest. F.J. has received grant/research support from the Brain and Behaviour Research Institute, the National Health and Medical Research Council (NHMRC), Australian Rotary Health, the Geelong Medical Research Foundation, the Ian Potter Foundation, Eli Lilly, Meat and Livestock Australia, Woolworths Limited, Fernwood Gyms, The Wilson Foundation, GMHBA, and the University of Melbourne and has received speakers honoraria from Sanofi-Synthelabo, Janssen Cilag, Servier, Pfizer, Health Ed, Network Nutrition, Angelini Farmaceutica, Eli Lilly, and Metagenics. O.M.D. is an R.D. Wright Biomedical Research Fellow and has received grant support from the Brain and Behavior Foundation, Simons Autism Foundation, Stanley Medical Research Institute, Deakin University, Lilly, NHMRC, and ASBDD/ Servier. She has also received in kind support from BioMedica Nutracuticals, Nutrition Care and Bioceuticals. All other authors have no relevant interests to declare.

\section{Supporting Information}

The following Supporting Information is available through the online version of this article at the publisher's website.

Appendix S1 PRISMA checklist

Appendix S2 Risk-of-bias summaries for included studies

\section{Appendix S3 Forest-plot of publication bias}

\section{REFERENCES}

1. Maes M, Leonard B, Fernandez A, et al. (Neuro)inflammation and neuroprogression as new pathways and drug targets in depression: from antioxidants to kinase inhibitors. Prog Neuropsychopharmacol Biol Psychiatry. 2011;35:659-663.

2. Miller $\mathrm{AH}$, Raison $\mathrm{CL}$. The role of inflammation in depression: from evolutionary imperative to modern treatment target. Nat Rev Immunol. 2015;16:22-34.

3. Moylan S, Maes M, Wray NR, et al. The neuroprogressive nature of major depressive disorder: pathways to disease evolution and resistance, and therapeutic implications. Mol Psychiatry. 2013;18:595-606.

4. Lopresti AL, Drummond PD. Saffron (Crocus sativus) for depression: a systematic review of clinical studies and examination of underlying antidepressant mechanisms of action. Hum Psychopharmacol Clin Exp. 2014;29:517-527.

5. Ettehadi H, Mojabi SN, Ranjbaran M, et al. Aqueous extract of saffron (Crocus sativus) increases brain dopamine and glutamate concentrations in rats. JBBS. 2013;3:315-319.

6. Georgiadou G, Tarantilis PA, Pitsikas N. Effects of the active constituents of Crocus sativus L., crocins, in an animal model of obsessive-compulsive disorder. Neurosci Lett. 2012;528:27-30.

7. Razavi $\mathrm{M}$, Hosseinzadeh $\mathrm{H}$, Abnous $\mathrm{K}$, et al. Crocin restores hypotensive effect of subchronic administration of diazinon in rats. Iran J Basic Med Sci. 2013;16:64-72.

8. Boskabady MH, Farkhondeh T. Antiinflammatory, antioxidant, and immunomodulatory effects of Crocus sativus $L$. and its main constituents. Phytother Res. 2016;30:1072-1094

9. Samarghandian S, Nezhad MA, Samini F, et al. The role of saffron in attenuating age-related oxidative damage in rat hippocampus. Recent Pat Food Nutr Agric 2017;8:183-189.

10. Poma A, Fontecchio G, Carlucci G, et al. Anti-inflammatory properties of drugs from saffron crocus. Antiinflamm Antiallergy Agents Med Chem. 2012;11:37-51.

11. Boskabady MH, Seyedhosseini Tamijani SM, et al. The effect of Crocus sativus extract on human lymphocytes' cytokines and T helper $2 / T$ helper 1 balance. J Med Food. 2011;14:1538-1545.

12. Halataei BA, Khosravi M, Arbabian S, et al. Saffron (Crocus sativus) aqueous extract and its constituent crocin reduces stress-induced anorexia in mice. Phytother Res. 2011;25:1833-1838.

13. Hooshmandi Z, Rohani AH, Eidi A, et al. Reduction of metabolic and behavioral signs of acute stress in male Wistar rats by saffron water extract and its constituent safranal. Pharm Biol. 2011:49:947-954.

14. Vahdati Hassani F, Naseri V, Razavi BM, et al. Antidepressant effects of crocin and its effects on transcript and protein levels of CREB, BDNF, and VGF in rat hippocampus. Daru. 2014;22:16

15. Ghasemi T, Abnous K, Vahdati F, et al. Antidepressant effect of Crocus sativus aqueous extract and its effect on CREB, BDNF, and VGF transcript and protein levels in rat hippocampus. Drug Res (Stuttg). 2015;65:337-343.

16. Hausenblas HA, Saha D, Dubyak PJ, et al. Saffron (Crocus sativus L.) and major depressive disorder: a meta-analysis of randomized clinical trials. J Integr Med. 2013;11:377-383.

17. Yang $X$, Chen $X, F u$, et al. Comparative efficacy and safety of Crocus sativus $L$. for treating mild to moderate major depressive disorder in adults: a metaanalysis of randomized controlled trials. Neuropsychiatr Dis Treat. 2018;14:1297-1305.

18. Liberati A, Altman DG, Tetzlaff J, et al. The PRISMA statement for reporting systematic reviews and meta-analyses of studies that evaluate health care interventions: explanation and elaboration. PLoS Med. 2009;6:e1000100.

19. Jadad AR, Moore RA, Carroll D, et al. Assessing the quality of reports of randomized clinical trials: is blinding necessary? Control Clin Trials. 1996;17:1-12.

20. Comprehensive Meta-analysis Version 3 [Computer Program]. Englewood, NJ: Biostat 104; 2011.

21. DerSimonian R, Laird N. Meta-analysis in clinical trials. Control Clin Trials. 1986;7:177-188.

22. Abedimanesh N, Ostadrahimi A, Bathaie $\mathrm{SZ}$, et al. Effects of saffron aqueous extract and its main constituent, crocin, on health-related quality of life, depression, and sexual desire in coronary artery disease patients: a double-blind, placebo-controlled, randomized clinical trial. Iran Red Crescent Med J. 2017;19:e13676. 
23. Akhondzadeh Basti A, Moshiri E, Noorbala AA, et al. Comparison of petal of Crocus sativus $L$. and fluoxetine in the treatment of depressed outpatients: a pilot double-blind randomized trial. Prog Neuropsychopharmacol Biol Psychiatry. 2007;31:439-442.

24. Akhondzadeh S, Tahmacebi-Pour N, Noorbala A, et al. Crocus sativus L. in the treatment of mild to moderate depression: a double-blind, randomized and placebocontrolled trial. Phytother Res. 2005;19:148-151.

25. Akhondzadeh S, Fallah-Pour H, Afkham K, et al. Comparison of Crocus sativus L. and imipramine in the treatment of mild to moderate depression: a pilot doubleblind randomized trial [ISRCTN45683816]. BMC Complement Altern Med. 2004;4:12.

26. Agha-Hosseini M, Kashani L, Aleyaseen A, et al. Crocus sativus L. (saffron) in the treatment of premenstrual syndrome: a double-blind, randomised and placebocontrolled trial. BJOG. 2008;115:515-519.

27. Ghajar A, Neishabouri SM, Velayati N, et al. Crocus sativus L versus citalopram in the treatment of major depressive disorder with anxious distress: a double-blind, controlled clinical trial. Pharmacopsychiatry. 2017;50:152-160.

28. Jafarnia N, Ghorbani Z, Nokhostin M, et al. Effect of saffron (Crocus sativus L.) as an add-on therapy to sertraline in mild to moderate generalized anxiety disorder: a double blind randomized controlled trial. Arch Neurosci. 2017;4:e14332.

29. Jam IN, Sahebkar AH, Eslami S, et al. The effects of crocin on the symptoms of depression in subjects with metabolic syndrome. Adv Clin Exp Med. 2017;26:925-930.

30. Jelodar G, Javid Z, Sahraian A, et al. Saffron improved depression and reduced homocysteine level in patients with major depression: a randomized, double-blind study. Avicenna J Phytomed. 2018;8:43-50.

31. Kashani L, Esalatmanesh S, Eftekhari F, et al. Efficacy of Crocus sativus (saffron) in treatment of major depressive disorder associated with post-menopausal hot flashes: a double-blind, randomized, placebo-controlled trial. Arch Gynecol Obstet. 2018;297:717-724.

32. Kashani L, Eslatmanesh $\mathrm{S}$, Saedi $\mathrm{N}$, et al. Comparison of saffron versus fluoxetine in treatment of mild to moderate postpartum depression: a double-blind, randomized clinical trial. Pharmacopsychiatry. 2017;50:64-68.

33. Kashani L, Raisi F, Saroukhani S, et al. Saffron for treatment of fluoxetine-induced sexual dysfunction in women: randomized double-blind placebo-controlled study. Hum Psychopharmacol Clin Exp. 2013;28:54-60.

34. Kell G, Beccaria G, Rao A, et al. Affron ${ }^{\circledR}$ a novel saffron extract (Crocus sativus L.) improves mood in healthy adults over 4 weeks in a double-blind, parallel, randomized, placebo-controlled clinical trial. Complement Ther Med. 2017;33:58-64.

35. Lopresti AL, Drummond PD, Inarejos-Garcia AM, et al. Affron ${ }^{\circledR}$, a standardised extract from saffron (Crocus sativus $L$.) for the treatment of youth anxiety and depressive symptoms: a randomised, double-blind, placebo-controlled study. J Affect Disord. 2018;232:349-357.
36. Mazidi M, Shemshian M, Mousavi SH, et al. A double-blind, randomized and placebo-controlled trial of saffron (Crocus sativus $L$.) in the treatment of anxiety and depression. J Complement Integr Med. 2016;13:195-199.

37. Moazen-Zadeh E, Abbasi SH, Safi-Aghdam H, et al. Effects of saffron on cognition, anxiety, and depression in patients undergoing coronary artery bypass grafting: a randomized double-blind placebo-controlled trial. J Altern Complement Med. 2018;24:361-368.

38. Modabbernia A, Sohrabi $\mathrm{H}$, Nasehi A, et al. Effect of saffron on fluoxetine-induced sexual impairment in men: randomized double-blind placebo-controlled trial. Psychopharmacology. 2012;223:381-388.

39. Moshiri E, Basti A, Noorbala A, et al. Crocus sativus L. (petal) in the treatment of mild-to-moderate depression: a double-blind, randomized and placebocontrolled trial. Phytomedicine. 2006;13:607-611.

40. Noorbala A, Akhondzadeh S, Tahmacebi-Pour N, et al. Hydro-alcoholic extract of Crocus sativus $L$. versus fluoxetine in the treatment of mild to moderate depression: a double-blind, randomized pilot trial. J Ethnopharmacol. 2005;97:281-284.

41. Sahraian A, Jelodar S, Javid Z, et al. Study the effects of saffron on depression and lipid profiles: a double blind comparative study. Asian J Psychiatry. 2016;22:174-176.

42. Shahmansouri N, Farokhnia M, Abbasi S, et al. A randomized, double-blind, clinical trial comparing the efficacy and safety of Crocus sativus $L$. with fluoxetine for improving mild to moderate depression in post percutaneous coronary intervention patients. J Affect Disord. 2014;155:216-222.

43. Tabeshpour J, Sobhani F, Sadjadi SA, et al. A double-blind, randomized, placebocontrolled trial of saffron stigma (Crocus sativus $L$.) in mothers suffering from mildto-moderate postpartum depression. Phytomedicine. 2017;36:145-152.

44. Talaei A, Hassanpour Moghadam M, Sajadi Tabassi SA, et al. Crocin, the main active saffron constituent, as an adjunctive treatment in major depressive disorder: a randomized, double-blind, placebo-controlled, pilot clinical trial. J Affect Disord. 2015;174:51-56.

45. Modaghegh MH, Shahabian M, Esmaeili HA, et al. Safety evaluation of saffron (Crocus sativus) tablets in healthy volunteers. Phytomedicine. 2008;15:1032-1037.

46. Cipriani A, Furukawa TA, Salanti G, et al. Comparative efficacy and acceptability of 21 antidepressant drugs for the acute treatment of adults with major depressive disorder: a systematic review and network meta-analysis. Lancet (London, England). 2018;391:1357-1366.

47. Moosavi S, Ahmadi M, Amini M, et al. The effects of 40 and $80 \mathrm{mg}$ hydro- alcoholic extract of crocus sativus in the treatment of mild to moderate depression. $J$ Mazandaran University Med Sci. 2014;24:47-53.

48. Rapaport MH, Nierenberg AA, Schettler PJ, et al. Inflammation as a predictive biomarker for response to omega-3 fatty acids in major depressive disorder: a proof of concept study. Mol Psychiatry. 2016;21:71-79. 